\title{
The probability that random positive integers are $k$-wise relatively prime
}

\author{
Jerry Hu
}

\begin{abstract}
The positive integers $a_{1}, a_{2}, \ldots, a_{s}$ are $k$-wise relatively prime if any $k$ of them are relatively prime. Exact formula is obtained for the probability that $\mathrm{s}$ positive integers are $k$-wise relatively prime.
\end{abstract}

A classic result in number theory is that the probability that two given integers are relatively prime is $6 / \pi^{2}$. More generally the probability that $s$ positive integers chosen arbitrarily and independently are relatively prime is $1 / \zeta(s)$, where $\zeta(s)$ is Riemann's zeta function. A short accessible proof of this result is in the paper of Nymann [1]. Recently, in 2002 Tóth [2] solved the problem of finding the probability that $s$ positive integers are pairwise relatively prime by the recursion method.

The positive integers $a_{1}, a_{2}, \ldots, a_{s}$ are $k$-wise relatively prime if any $k$ of them are relatively prime and are $k$-wise relatively prime to $a$ if gcd of any $k$ of them is relatively prime to $a$. In this note we consider the problem of finding the probability $A_{s, k}$ that s positive integers are $k$-wise relatively prime.

For a $(k-1)$-tuple of positive integers $u=\left(u_{1}, \ldots, u_{k-1}\right)$, let $Q_{s, k}^{(u)}(n)$ denote the number of $s$-tuples of positive integers $a_{1}, a_{2}, \ldots, a_{s}$ with $1 \leq a_{1}, a_{2}, \ldots, a_{s} \leq n$ such that $a_{1}, a_{2}, \ldots, a_{s}$ are $k$-wise relatively prime and are $i$-wise relatively prime to $u_{i}$ for $i=1,2, \ldots, k-1$.

The next theorem gives an asymptotic formula for $Q_{s, k}^{(u)}(n)$ and the exact values of $A_{s, k}$.

Theorem 1 For fixed $s \geq 1, k \geq 2$, we have uniformly for $n, u_{i} \geq 1$ with $\left(u_{i}, u_{j}\right)=1$ for $1 \leq i \neq j \leq k-1$,

$$
Q_{s, k}^{(u)}(n)=A_{s, k} \prod_{i=1}^{k-1} f_{s, k, i}\left(u_{i}\right) n^{s}+O\left(\theta\left(u_{1}\right) n^{s-1} \log ^{\delta(s, k)} n\right),
$$

where

$$
A_{s, k}=\prod_{p}\left(1-\frac{1}{p}\right)^{s-k+1} \sum_{m=0}^{k-1}\left(\begin{array}{c}
s \\
m
\end{array}\right)\left(1-\frac{1}{p}\right)^{k-1-m} \frac{1}{p^{m}},
$$




$$
\begin{gathered}
f_{s, k, i}\left(u_{i}\right)=\prod_{p \mid u_{i}}\left(1-\frac{\sum_{m=i}^{k-1}\left(\begin{array}{c}
s \\
m
\end{array}\right)(p-1)^{k-1-m}}{\sum_{m=0}^{k-1}\left(\begin{array}{c}
s \\
m
\end{array}\right)(p-1)^{k-1-m}}\right), \\
\delta(s, k)=\max \left\{\left(\begin{array}{c}
s-1 \\
i
\end{array}\right), i=1,2, \ldots, k-1\right\},
\end{gathered}
$$

and $\theta\left(u_{1}\right)$ is the number of squarefree divisors of $u_{1}$.

Note that $f_{s, k, i}\left(u_{i}\right)$ is a multiplicative arithmetic function for $i=1, \ldots, k-1$.

Corollary 2 The probability that $s$ positive integers $a_{1}, a_{2}, \ldots, a_{s}$ are $k$-wise relatively prime and are $i$-wise relatively prime to $u_{i}$ for $i=1,2, \ldots, k-1$ is

$$
\lim _{n \rightarrow \infty} \frac{Q_{s, k}^{(u)}(n)}{n^{s}}=A_{s, k} \prod_{i=1}^{k-1} f_{s, k, i}\left(u_{i}\right) .
$$

For $u_{1}=u_{2}=\cdots=u_{k-1}=1$, the probability that $s$ positive integers are $k$-wise relatively prime is

$$
A_{s, k}=\prod_{p}\left(1-\frac{1}{p}\right)^{s-k+1} \sum_{m=0}^{k-1}\left(\begin{array}{c}
s \\
m
\end{array}\right)\left(1-\frac{1}{p}\right)^{k-1-m} \frac{1}{p^{m}} .
$$

For a pair of positive integers $a$ and $b$, let $(a, b]$ denote the product of prime powers $p^{e}$ such that $p^{e}$ divides $b$, but $p^{e+1}$ does not divide $b$, and $p$ also divides $a$. Note that $(a, b)$ and $b /(a, b)$ may not be relatively prime, but $(a, b)$ and $b /(a, b]$ are relatively prime.

To prove the theorem we need the following lemmas.

Lemma 3 For $s, n \geq 1, k \geq 2$, and $u=\left(u_{1}, u_{2}, \ldots, u_{k-1}\right)$ with $u_{1}, \ldots, u_{k-1} \geq 1$ and $\left(u_{i}, u_{j}\right)=1$ for $i \neq j$,

$$
Q_{s+1, k}^{(u)}(n)=\sum_{\substack{j=1 \\\left(j, u_{1}\right)=1}}^{n} Q_{s, k}^{(j * u)}(n),
$$

where

$$
j * u=\left(u_{1}\left(j, u_{2}\right), \frac{u_{2}\left(j, u_{3}\right)}{\left(j, u_{2}\right]}, \ldots, \frac{u_{k-2}\left(j, u_{k-1}\right)}{\left(j, u_{k-2}\right]}, \frac{j u_{k-1}}{\left(\prod_{i=2}^{k-1}\left[j, u_{i}\right)\right)\left(j, u_{k-1}\right]}\right) .
$$

Proof. An $(s+1)$-tuple of positive integers $a_{1}, a_{2}, \ldots, a_{s+1}$ are $k$-wise relatively prime and are $i$-wise relatively prime to $u_{i}$ for $i=1,2, \ldots, k-1$ if and only if the first $s$-tuple of positive integers $a_{1}, a_{2}, \ldots, a_{s}$ are $k$-wise relatively prime and 
are $i$-wise relatively prime to $u_{i}$ and $\left(a_{s+1}, u_{i+1}\right)$ for $i=1,2, \ldots, k-2$ and are $(k-1)$-wise relatively prime to $u_{k-1}$ and $a_{s+1}$, and $\left(a_{s+1}, u_{1}\right)=1$, we have

$$
Q_{s+1, k}^{(u)}(n)=\sum_{\substack{a_{s+1}=1 \\\left(a_{s+1}, u_{1}\right)=1}}^{n} Q_{s, k}^{\left(a_{s+1} *^{\prime} u\right)}(n)=\sum_{\substack{j=1 \\\left(j, u_{1}\right)=1}}^{n} Q_{s, k}^{\left(j *^{\prime} u\right)}(n),
$$

where

$$
j *^{\prime} u=\left(u_{1}\left(j, u_{2}\right), u_{2}\left(j, u_{3}\right), \ldots, u_{k-2}\left(j, u_{k-1}\right), j u_{k-1}\right) .
$$

Note that the $(k-1)$-tuples of positive integers $j *^{\prime} u$ are not pairwise relatively prime, so Theorem 1 can not be applied to $Q_{s, k}^{\left(j *^{\prime} u\right)}(n)$ and the above recurrence relation can not be used in the proof of Theorem 1 by induction.

To complete the proof of the lemma, it suffices to show that $Q_{s, k}^{\left(j *^{\prime} u\right)}(n)=$ $Q_{s, k}^{(j * u)}(n)$. For an $s$-tuple of positive integers, if each of them is relatively prime to $\left(j, u_{2}\right)$, then they are pairwise relatively prime to $\left(j, u_{2}\right)$, and pairwise relatively prime to $\left(j, u_{2}\right]$ since $\left(j, u_{2}\right)$ and $\left(j, u_{2}\right]$ contain the same prime factors, thus they are pairwise relatively prime to $u_{2}\left(j, u_{3}\right)$ if and only if they are pairwise relatively prime to $u_{2}\left(j, u_{3}\right) /\left(j, u_{2}\right]$. Similarly, for $i=3,4, \ldots, k-2$, if an $s$-tuple of positive integers are $(i-1)$-wise relatively prime to $\left(j, u_{i}\right)$, then they are $i$-wise relatively prime to $u_{i}\left(j, u_{i+1}\right)$ if and only if they are $i$-wise relatively prime to $u_{i}\left(j, u_{i+1}\right) /\left(j, u_{i}\right]$. For $i=1,2, \ldots, k-2$, if an $s$-tuple of positive integers are $i$-wise relatively prime to $\left(j, u_{i+1}\right)$, then they are $(k-1)$-wise relatively prime to $\left[j, u_{i+1}\right)$, and $(k-1)$-wise relatively prime to $\left(j, u_{k-1}\right]$, thus they are $(k-1)$-wise relatively prime to $j u_{k-1}$ if and only if they are $(k-1)$-wise relatively prime to $j u_{k-1} /\left(\prod_{i=2}^{k-1}\left[j, u_{i}\right)\right)\left(j, u_{k-1}\right]$. Therefore, $Q_{s, k}^{\left(j *^{\prime} u\right)}(n)=Q_{s, k}^{(j * u)}(n)$.

Note that the $(k-1)$-tuple of integers in $j * u$ are pairwise relatively prime, so we can apply Theorem 1 to $Q_{s, k}^{(j * u)}(n)$ in the proof of Theorem 1 by induction.

Lemma 4 For $k, u_{i} \geq 1$,

$$
\begin{gathered}
\frac{f_{s, k, i}\left(u_{i}\right)}{f_{s, k, i+1}\left(u_{i}\right)}=\sum_{d \mid u_{i}} \frac{\mu(d)\left(\begin{array}{c}
s \\
i
\end{array}\right)^{\omega(d)}}{\alpha_{s, k, i}(d)}, i=1,2, \ldots, k-2, \\
f_{s, k, k-1}\left(u_{k-1}\right)=\sum_{d \mid u_{k-1}} \frac{\mu(d)\left(\begin{array}{c}
s \\
k-1
\end{array}\right)^{\omega(d)}}{\alpha_{s, k, k-1}(d)},
\end{gathered}
$$

where

$$
\alpha_{s, k, i}(d)=d^{i} \prod_{p \mid d} \sum_{m=0}^{i}\left(\begin{array}{c}
s \\
m
\end{array}\right)\left(1-\frac{1}{p}\right)^{i-m} \frac{1}{p^{m}}, i=1,2, \ldots, k-1,
$$

and $\omega\left(u_{i}\right)$ denote the number of distinct prime factors of $u_{i}$. 
Proof. Since both $\frac{f_{s, k, i}\left(u_{i}\right)}{f_{s, k, i+1}\left(u_{i}\right)}$ and $f_{s, k, k-1}\left(u_{k-1}\right)$ are multiplicative arithmetic functions, it suffices to verify for $u_{i}=p^{a}$ a prime power:

$$
\begin{gathered}
\sum_{d \mid p^{a}} \frac{\mu(d)\left(\begin{array}{c}
s \\
i
\end{array}\right)^{\omega(d)}}{\alpha_{s, k, i}(d)}=1-\left(\begin{array}{c}
s \\
i
\end{array}\right)\left(\sum_{m=0}^{i}\left(\begin{array}{c}
s \\
m
\end{array}\right)(p-1)^{i-m}\right)^{-1}=\frac{f_{s, k, i}\left(p^{a}\right)}{f_{s, k, i+1}\left(p^{a}\right)}, \\
\sum_{d \mid p^{a}} \frac{\mu(d)\left(\begin{array}{c}
s \\
k-1
\end{array}\right)^{\omega(d)}}{\alpha_{s, k, k-1}(d)}=1-\left(\begin{array}{c}
s \\
k-1
\end{array}\right)\left(\sum_{m=0}^{k-1}\left(\begin{array}{c}
s \\
m
\end{array}\right)(p-1)^{k-1-m}\right)^{-1}=f_{s, k, k-1}\left(p^{a}\right) .
\end{gathered}
$$

For the proof of the theorem, we proceed by induction on $s$. For $s=1$, we have by the Inclusion-Exclusion Principle

$$
\begin{aligned}
Q_{1, k}^{(u)}(n) & =\sum_{\substack{j=1 \\
\left(j, u_{1}\right)=1}}^{n} 1=\sum_{d \mid u_{1}} \mu(d)\left\lfloor\frac{n}{d}\right\rfloor=\sum_{d \mid u_{1}} \mu(d)\left(\frac{n}{d}+O(1)\right) \\
& =n \sum_{d \mid u_{1}} \frac{\mu(d)}{d}+O\left(\sum_{d \mid u_{1}} \mu^{2}(d)\right) .
\end{aligned}
$$

Hence,

$$
Q_{1, k}^{(u)}(n)=\sum_{\substack{j=1 \\\left(j, u_{1}\right)=1}}^{n} 1=n \frac{\phi\left(u_{1}\right)}{u_{1}}+O\left(\theta\left(u_{1}\right)\right)
$$

and (11) is true for $s=1$ with $A_{1, k}=1, f_{1, k, i}\left(u_{i}\right)=1$ for $i \geq 2$, and $f_{1, k, 1}\left(u_{1}\right)=$ $\frac{\phi\left(u_{1}\right)}{u_{1}}, \phi$ denoting the Euler function.

Suppose that (1) is valid for $s$, we prove it for $s+1$. From Lemma 3, we have

$$
\begin{aligned}
Q_{s+1, k}^{(u)}(n) & =\sum_{\substack{j=1 \\
\left(j, u_{1}\right)=1}}^{n} Q_{s, k}^{(j * u)}(n) \\
& =\sum_{\substack{j=1 \\
\left(j, u_{1}\right)=1}}^{n} A_{s, k} \prod_{i=1}^{k-2} f_{s, k, i}\left(\frac{u_{i}\left(j, u_{i+1}\right)}{\left(j, u_{i}\right]}\right) f_{s, k, k-1}\left(\frac{j u_{k-1}}{\left(\prod_{i=2}^{k-1}\left[j, u_{i}\right)\right)\left(j, u_{k-1}\right]}\right) n^{s} \\
& +O\left(\theta\left(u_{1}\left(j, u_{2}\right)\right) n^{s-1} \log ^{\delta(s, k)} n\right) \\
= & A_{s, k} \prod_{i=1}^{k-1} f_{s, k, i}\left(u_{i}\right) n^{s} \sum_{\substack{j=1 \\
\left(j, u_{1}\right)=1}}^{n} \prod_{i=1}^{k-2} \frac{f_{s, k, i}\left(\left(j, u_{i+1}\right)\right)}{f_{s, k, i+1}\left(\left(j, u_{i+1}\right)\right)} f_{s, k, k-1}\left(\frac{j}{\prod_{i=2}^{k-1}\left[j, u_{i}\right)}\right)
\end{aligned}
$$




$$
+O\left(\theta\left(u_{1}\right) n^{s-1} \log ^{\delta(s, k)} n \sum_{j=1}^{n} \theta(j)\right) .
$$

Here $\sum_{j=1}^{n} \theta(j) \leq \sum_{j=1}^{n} \tau_{2}(j)=O(n \log n)$, where $\tau_{2}=\tau$ is the divisor function.

Furthermore, from Lemma 4,

$$
\begin{aligned}
& \sum_{\substack{j=1 \\
\left(j, u_{1}\right)=1}}^{n} \prod_{i=1}^{k-2} \frac{f_{s, k, i}\left(\left(j, u_{i+1}\right)\right)}{f_{s, k, i+1}\left(\left(j, u_{i+1}\right)\right)} f_{s, k, k-1}\left(\frac{j}{\prod_{i=2}^{k-1}\left[j, u_{i}\right)}\right) \\
& =\sum_{\substack{j=1 \\
\left(j, u_{1}\right)=1}}^{n} \prod_{i=1}^{k-2} \sum_{d_{i} \mid\left(j, u_{i+1}\right)} \frac{\mu\left(d_{i}\right)\left(\begin{array}{c}
s \\
i
\end{array}\right)^{\omega\left(d_{i}\right)}}{\alpha_{s, k, i}\left(d_{i}\right)} \sum_{d_{k-1} \mid \frac{j}{\prod_{i=2}^{k-1}\left[j, u_{i}\right)}} \frac{\mu\left(d_{k-1}\right)\left(\begin{array}{c}
s \\
k-1
\end{array}\right)^{\omega\left(d_{k-1}\right)}}{\alpha_{s, k, k-1}\left(d_{k-1}\right)} \\
& =\sum_{\substack{\left.d_{1} d_{2} \ldots d_{k-1} e=j \leq n \\
d_{i}\left|\left(j, u_{i+1}\right), i=1, \ldots, k-2 \\
d_{k-1}\right| j, u_{2}\right) \ldots\left[j, u_{k-1}\right) \\
\left(j, u_{1}\right)=1}} \prod_{i=1}^{k-1} \frac{\mu\left(d_{i}\right)\left(\begin{array}{l}
s \\
i
\end{array}\right)^{\omega\left(d_{i}\right)}}{\alpha_{s, k, i}\left(d_{i}\right)} \\
& =\sum_{\substack{d_{1} d_{2} \ldots d_{k-1} \leq n \\
d_{i} \mid u_{i+1}, i=1, \ldots, k-2 \\
\left(d_{k-1}, u_{i}\right)=1, i=1,2, \ldots, k-1}} \prod_{i=1}^{k-1} \frac{\mu\left(d_{i}\right)\left(\begin{array}{l}
s \\
i
\end{array}\right)^{\omega\left(d_{i}\right)}}{\alpha_{s, k, i}\left(d_{i}\right)} \sum_{\substack{n \\
e \leq \frac{n}{d_{1} d_{2} \cdots d_{k-1}} \\
\left(e, u_{1}\right)=1}} 1
\end{aligned}
$$

Using (2), we have

$$
\begin{aligned}
& \sum_{\substack{j=1 \\
\left(j, u_{1}\right)=1}}^{n} \prod_{i=1}^{k-2} \frac{f_{s, k, i}\left(\left(j, u_{i+1}\right)\right)}{f_{s, k, i+1}\left(\left(j, u_{i+1}\right)\right)} f_{s, k, k-1}\left(\frac{j}{\prod_{i=2}^{k-1}\left[j, u_{i}\right)}\right) \\
& =\sum_{\substack{d_{1} d_{2} \ldots d_{k-1}=d \leq n \\
d_{i} \mid u_{i+1}, i=1, \ldots, k-2 \\
\left(d_{k-1}, u_{i}\right)=1, i=1,2, \ldots, k-1}} \prod_{i=1}^{k-1} \frac{\mu\left(d_{i}\right)\left(\begin{array}{c}
s \\
i
\end{array}\right)^{\omega\left(d_{i}\right)}}{\alpha_{s, k, i}\left(d_{i}\right)}\left(\frac{\phi\left(u_{1}\right)}{u_{1}} \frac{n}{d_{1} d_{2} \cdots d_{k-1}}+O\left(\theta\left(u_{1}\right)\right)\right) \\
& =\frac{\phi\left(u_{1}\right)}{u_{1}} n \sum_{\substack{d_{1} d_{2} \ldots d_{k-1}=d \leq n \\
d_{i} \mid u_{i+1}, i=1, \ldots, k-2 \\
\left(d_{k-1}, u_{i}\right)=1, i=1,2, \ldots, k-1}} \prod_{i=1}^{k-1} \frac{\mu\left(d_{i}\right)\left(\begin{array}{l}
s \\
i
\end{array}\right)^{\omega\left(d_{i}\right)}}{d_{i} \alpha_{s, k, i}\left(d_{i}\right)} \\
& +O\left(\theta\left(u_{1}\right) \sum_{d \leq n} \frac{\delta(s+1, k)^{\omega(d)}}{d}\right)
\end{aligned}
$$


since $\alpha_{s, k, i}\left(d_{i}\right)>d_{i}$.

Hence, the main term of (5) is

$$
\begin{aligned}
& \frac{\phi\left(u_{1}\right)}{u_{1}} n \sum_{\substack{d_{i} \mid u_{i+1}, i=1, \ldots, k-2 \\
\left(d_{k-1}, u_{i}\right)=1, i=1,2, \ldots, k-1}} \prod_{i=1}^{k-1} \frac{\mu\left(d_{i}\right)\left(\begin{array}{l}
s \\
i
\end{array}\right)^{\omega\left(d_{i}\right)}}{d_{i} \alpha_{s, k, i}\left(d_{i}\right)} \\
& =\frac{\phi\left(u_{1}\right)}{u_{1}} n \prod_{i=2}^{k-1} \prod_{p \mid u_{i}}\left(1-\frac{\left(\begin{array}{c}
s \\
i-1
\end{array}\right)}{p \sum_{m=0}^{i-1}\left(\begin{array}{c}
s \\
m
\end{array}\right)(p-1)^{i-1-m}}\right) \prod_{p \nmid u_{1} \cdots u_{k-1}}\left(1-\frac{\left(\begin{array}{c}
s \\
k-1
\end{array}\right)}{p \sum_{m=0}^{k-1}\left(\begin{array}{c}
s \\
m
\end{array}\right)(p-1)^{k-1-m}}\right) \\
& =n \prod_{i=1}^{k-1} \prod_{p \mid u_{i}}\left(1-\frac{\left(\begin{array}{c}
s \\
i-1
\end{array}\right)}{p \sum_{m=0}^{i-1}\left(\begin{array}{c}
s \\
m
\end{array}\right)(p-1)^{i-1-m}}\right)\left(1-\frac{\left(\begin{array}{c}
s \\
k-1
\end{array}\right)}{p \sum_{m=0}^{k-1}\left(\begin{array}{c}
s \\
m
\end{array}\right)(p-1)^{k-1-m}}\right)^{-1} \\
& \prod_{p}\left(1-\frac{\left(\begin{array}{c}
s \\
k-1
\end{array}\right)}{p \sum_{m=0}^{k-1}\left(\begin{array}{c}
s \\
m
\end{array}\right)(p-1)^{k-1-m}}\right) \text {, }
\end{aligned}
$$

and its O-terms are

$$
\begin{aligned}
O\left(n \sum_{d>n} \frac{\delta(s+1, k)^{\omega(d)}}{d^{2}}\right) & =O\left(n \sum_{d>n} \frac{\tau_{\delta(s+1, k)}(d)}{d^{2}}\right) \\
& =O\left(\log ^{\delta(s+1, k)-1} n\right)
\end{aligned}
$$

by Lemma 3(b) in [2], which gives an asymptotic estimate of the sum

$$
\sum_{n>x} \frac{\tau_{k}(n)}{n^{2}}=O\left(\frac{\log ^{k-1} x}{x}\right)
$$

and

$$
\begin{aligned}
O\left(\theta\left(u_{1}\right) \sum_{d \leq n} \frac{\delta(s+1, k)^{\omega(d)}}{d}\right) & =O\left(\theta\left(u_{1}\right) \sum_{d \leq n} \frac{\tau_{\delta(s+1, k)}(d)}{d}\right) \\
& =O\left(\theta\left(u_{1}\right) \log ^{\delta(s+1, k)} n\right)
\end{aligned}
$$

from Lemma 3(a) in [2, which gives an asymptotic estimate of the sum

$$
\sum_{n \leq x} \frac{\tau_{k}(n)}{n}=O\left(\log ^{k} x\right) .
$$


Substituting into (3), we get

$$
\begin{aligned}
Q_{s+1, k}^{(u)}(n) & =A_{s, k} \prod_{p}\left(1-\frac{\left(\begin{array}{c}
s \\
k-1
\end{array}\right)}{p \sum_{m=0}^{k-1}\left(\begin{array}{c}
s \\
m
\end{array}\right)(p-1)^{k-1-m}}\right) \\
\prod_{i=1}^{k-1} f_{s, k, i} & \prod_{p \mid u_{i}}\left(1-\frac{\left(\begin{array}{c}
s \\
i-1
\end{array}\right)}{p \sum_{m=0}^{i-1}\left(\begin{array}{c}
s \\
m
\end{array}\right)(p-1)^{i-1-m}}\right)\left(1-\frac{\left(\begin{array}{c}
s \\
k-1
\end{array}\right)}{p \sum_{m=0}^{k-1}\left(\begin{array}{c}
s \\
m
\end{array}\right)(p-1)^{k-1-m}}\right)^{s+1} \\
& +O\left(n^{s} \log ^{\delta(s+1, k)-1} n\right)+O\left(\theta\left(u_{1}\right) n^{s} \log ^{\delta(s+1, k)} n\right)+O\left(\theta\left(u_{1}\right) n^{s} \log ^{\delta(s, k)+1} n\right) \\
= & A_{s+1, k} \prod_{i=1}^{k-1} f_{s+1, k, i}\left(u_{i}\right) n^{s+1}+O\left(\theta\left(u_{1}\right) n^{s} \log ^{\delta(s+1, k)} n\right)
\end{aligned}
$$

by a simple computation, which shows that the formula is true for $s+1$ and we complete the proof.

Acknowledgement. I would like to thank Professor László Tóth for helpful discussions of the topics of this paper.

\section{References}

[1] J.E. Nymann, On the probability that $k$ positive integers are relatively prime, J. Number Theory 4 (1972), 469-473.

[2] L. Tóth, The probability that $k$ positive integers are pairwise relatively prime, Fibonacci Quart. 40 (2002), 13-18.

University of Houston-Victoria, Department of Mathematics and Computer Science, School of Arts \& Sciences, 14000 University Blvd, Sugar Land, TX, 77479

E-mail: huj@uhv.edu 